\title{
A two-step iterative method and its acceleration for outer inverses
}

\author{
SHWETABH SRIVASTAVA* and DHARMENDRA K GUPTA
}

Department of Mathematics, Indian Institute of Technology Kharagpur, Kharagpur 721802, India

e-mail: shwetabhiit@gmail.com

MS received 4 April 2016; revised 11 May 2016; accepted 11 May 2016

\begin{abstract}
A two-step iterative method and its accelerated version for approximating outer inverse $A_{T, S}^{(2)}$ of an arbitrary matrix $A$ are proposed. A convergence theorem for its existence is established. The rigorous error bounds are derived. Numerical experiments involving singular square, rectangular, random matrices and a sparse matrix obtained by discretization of the Poisson's equation are solved. Iterations count, computational time and the error bounds are used to measure the performance of our method. On comparing our results with those of other iterative methods, it is seen that significantly better performance is achieved. Thus, enhanced speed and accuracy from the computational points of view has resulted for our methodology.
\end{abstract}

Keywords. Random matrices; sparse matrices; Penrose equations; group inverse; Poisson's equation; outer inverse.

\section{Introduction}

Let $\mathbb{C}_{r}^{m \times n}$ be the set of $(m \times n)$ matrices of rank $r$ over the complex field $\mathbb{C}$. For $A \in \mathbb{C}^{m \times n}$, let $\|A\|_{2}, A^{*}, A^{t}, R(A)$ and $N(A)$ represent the matrix 2-norm, the conjugate transpose, the transpose, the range space and the null space, respectively. Further, $\rho(X)$ and $\lambda_{\max }(X)$ denote spectral radius and the maximal eigenvalue of a square matrix $X \in \mathbb{C}^{n \times n}$. One of the most important and challenging tasks in numerical analysis is finding efficient iteration for computing outer inverse $A_{T, S}^{(2)}$ and develop its convergence analysis, where $T$ and $S$ stand for the subspaces of $\mathbb{C}^{n}$ and $\mathbb{C}^{m}$, respectively. For any matrix $A \in \mathbb{C}^{m \times n}$, the matrix $P \in \mathbb{C}^{n \times m}$ such that $P A P=P, R(P)=T$ and $N(P)=S$ is known as outer inverse $A_{T, S}^{(2)}$ of $A$ with the prescribed range $T$ and null space $S$. It is unique and important as some of the most commonly used generalized inverses can be derived from it by means of appropriate choices of $T$ and $S$. For example, for $T=$ $R\left(A^{*}\right)$ and $S=N\left(A^{*}\right)$, we get the Moore-Penrose inverse $A^{\dagger}$ of $A \in \mathbb{C}^{m \times n}$ represented as $A_{T, S}^{(2)}=A^{\dagger}$. We define $A^{\dagger}$ as the unique matrix $X \in \mathbb{C}^{n \times m}$ such that $A X A=A, X A X=X$, $(A X)^{*}=A X,(X A)^{*}=X A$. For $T=R\left(A^{j}\right)$ and $S=N\left(A^{j}\right)$, where the index $j$ of $A \in \mathbb{C}^{n \times n}$ is defined as $\min _{j}\left\{j: \operatorname{rank}\left(A^{j+1}\right)=\operatorname{rank}\left(A^{j}\right)\right\}$, we get the Drazin inverse $A^{d}$ such that $A_{T, S}^{(2)}=A^{d}$. It is defined as the unique matrix $X \in \mathbb{C}^{n \times n}$ such that $A^{j} X A=A^{j}, X A X=X, A X=X A$. For $j=1, X$ is known as the group inverse $A_{g}$ of $A$. Many other

*For correspondence important generalized inverses such as, the weighted Moore-Penrose inverse $A_{M, N}^{\dagger}$, the Bott-Duffin inverse $A_{(L)}^{(-1)}$ and the generalized Bott-Duffin inverse $A_{(L)}^{(\dagger)}$ are also its special cases (see, [1]). A number of application areas (see [2-4]) that give rise to the computation of $A_{T, S}^{(2)}$ are statistics, optimization, multi-body system dynamics, differential algebraic equations, image processing, etc. In literature, many researchers [5-11] have proposed many direct and iterative methods for computing outer inverses. Some of the direct methods studied are usually based on the $Q R$ factorization [12], Cholesky factorization [13], and Gaussian elimination [14]. In [14], authors presented a direct method for computing outer inverse $A_{T, S}^{(2)}$ based on the choice of proper matrix $G$ and the Gauss-Jordan elimination of the augmented matrix $\left[G \mid I\right.$, where $G \in \mathbb{C}^{n \times m}$ be such that $R(G)=T$ and $N(G)=S$ and $I$ be the identity matrix of suitable order. Based on the full-rank $L U$ decomposition of an appropriate matrix $M$, a new direct method is developed in [15]. The most frequently used simple and numerically stable Newton's iterative method [16, 17] for approximat$\operatorname{ing} A_{T, S}^{(2)}$ is given for $k=0,1,2, \ldots$, by,

$$
X_{k+1}=X_{k}\left(2 I-A X_{k}\right),
$$

where $X_{0}$ be a suitably chosen initial matrix and $I$ denotes the identity matrix of appropriate order. A method based on splitting of matrices is described in [18]. A quadratically convergent iterative scheme proposed in [19] based on matrix equations $X A X=X$ and $(X A)^{*}=X A$ is given for $k=0,1,2 \ldots$, by 


$$
X_{k+1}=(1+\beta) X_{k}-\beta X_{k} A X_{k}, \beta \in(0,1] .
$$

Starting with $X_{0}=\alpha G$, where $\alpha, \beta$ are real constants and $G \in \mathbb{C}^{n \times m}$ is a chosen matrix of rank $s$ such that $0<s \leq r$, it converges to outer inverse $A_{R(G), N(G)}^{(2)}$. A higher-order iterative scheme investigated in [20] for $A_{T, S}^{(2)}$ for arbitrary positive integer $p \geq 2$ is given for $k=0,1,2, \ldots$, by

$X_{k}=\left[C_{p}^{1} I-C_{p}^{2} X_{k-1} A+\ldots+(-1)^{p-1} C_{p}^{p}\left(X_{k-1} A\right)^{p-1}\right] X_{k-1}$,

where $C_{n}^{i}=\frac{n !}{i !(n-i) !}, \quad i=0,1,2, \ldots, n$ denote the binomial coefficients. It was shown that starting with $X_{0}=\alpha G$, where $G \in C^{n \times m}$ satisfies $R(G)=T$ and $N(G)=S$ and $\alpha$ be a non-zero complex scalar, method (2) converges to $A_{T, S}^{(2)}$ if and only if $\rho\left(\alpha G A-P_{T}\right)<1$, where the transformation $P_{T}$ denotes the orthogonal projector on $T$. In [21], author studied a linear order iterative scheme for computing $A_{T, S}^{(2)}$, given for $k=0,1,2 \ldots$ by, $X_{k+1}=X_{k}+\beta G\left(I-A X_{k}\right)$ for $G \in C^{n \times m}$ satisfying $R(G) \subset T, N(G) \supset S$ and a non-zero real scalar $\beta$. It was shown that starting with any initial approximation $X_{0} \in C^{n \times m}$ satisfying $R\left(X_{0}\right) \subset T$, the sequence $\left\{X_{k}\right\}$ converges to $A_{T, S}^{(2)}$ if and only if $\rho\left(P_{T}-\beta G A\right)<1$. Its $p$ th order extension was also developed by him. An iterative algorithm to compute $A_{T, S}^{(2)}$ under the concepts of restricted inner product is presented in [22]. An algorithm to compute $A_{T, S}^{(2)}$ using successive matrix squaring (SMS) is presented in [23].

In this paper, a two-step iterative method and its accelerated version for approximating outer inverse $A_{T, S}^{(2)}$ of an arbitrary matrix $A$ are proposed. A convergence theorem for its existence is established. The rigorous error bounds are derived. Numerical experiments involving singular square, rectangular, random matrices and a sparse matrix obtained by discretization of the Poisson's equation are solved. Iterations count, computational time and the error bounds are used to measure the performance of our methods. On comparing our methods with others, it is seen that significantly better results are obtained. Thus, our method leads to enhanced speed and accuracy from the computational points of view.

The paper is organized as follows. section 1 is the introduction. In section 2, two-step iterative method for $A_{T, S}^{(2)}$ and some basic lemmas used are described. A convergence theorem along with its error bounds is also derived here. Two-step accelerated iterative method for its computation is established in section 3. A number of numerical experiments are performed in section 4. Finally, conclusions are included in section 5.

\section{Two-step iterative method and its convergence analysis}

In this section, a two-step iterative method for computing $A_{T, S}^{(2)}$ is described. Its convergence analysis and error bounds are also derived. Let $\operatorname{dim}(T)=\operatorname{dim}\left(S^{\perp}\right)=t \leq r$, where $S^{\perp}$ denotes the orthogonal complement of a subspace $S$. Let $G \in \mathbb{C}^{n \times m}$ satisfies $R(G) \subseteq T$ and $N(G) \supseteq S$. Starting with $X_{0}=\beta G$, for a non-zero real scalar $\beta$, the method is given for $k=0,1,2, \ldots$, by

$$
\begin{gathered}
Z_{k+1}=X_{k}+X_{k} R_{k}, \\
X_{k+1}=X_{k}+Z_{k+1} R_{k},
\end{gathered}
$$

where $R_{k}=I-A X_{k}$ and sequence $\left\{Z_{k+1}\right\}$ is approximated by the Newton's iteration. The following basic results are important to prove our further statements.

Lemma $2.1 \quad[16,24]$ Let $\operatorname{dim} T=\operatorname{dim} S^{\perp}=t \leq r$. A has a unique outer inverse $X$ for $R(X)=T$ and $N(X)=S$ if and only if

$$
A T \oplus S=\mathbb{C}^{m},
$$

where $\oplus$ denotes the direct sum of subspaces AT and $S$.

We have following remarks from Lemma 2.1.

Remark 2.1 The condition (5) is equivalent to the following conditions.

$$
\begin{aligned}
A^{*} S^{\perp} \oplus T^{\perp} & =\mathbb{C}^{n} . \\
P_{S^{\perp}} A T & =S^{\perp} . \\
P_{T} A^{*} S^{\perp} & =T .
\end{aligned}
$$

Remark 2.2 ([16, 25]) $A A_{T, S}^{(2)}=P_{A T, S} \quad$ and $\quad A_{T, S}^{(2)} A=$ $P_{T,\left(A^{*} S^{\perp}\right)^{\perp}}$.

Lemma 2.2 [16] For any $A \in \mathbb{C}^{m \times n}, P_{L, M} A=A$ if and only if $R(A) \subset L$ and $A P_{L, M}=A$ if and only if $N(A) \supset M$.

Lemma 2.3 The sequence $\left\{X_{k}\right\}$ for $k \geq 0$ from (3) to (4) with $X_{0}=\beta G$, where $G \in \mathbb{C}^{n \times m}$ be such that $R(G) \subseteq T$ and $N(G) \supseteq S$, satisfies (i) $A_{T, S}^{(2)} A X_{k}=X_{k}$ and (ii) $A A_{T, S}^{(2)} A X_{k}=$ $A X_{k}$.

Proof This lemma can be proved by mathematical induction. Clearly, (i) follows for $k=0$ from Remark 2.2 and Lemma 2.2 as

$$
A_{T, S}^{(2)} A X_{0}=P_{T,\left(A^{*} S^{\perp}\right)^{\perp}} X_{0}=\beta P_{T,\left(A^{*} S^{\perp}\right)^{\perp}} G=\beta G=X_{0} .
$$

Suppose $A_{T, S}^{(2)} A X_{k}=X_{k}$ holds for some positive integer $k$. To show that it holds for $k+1$, we consider 


$$
\begin{aligned}
A_{T, S}^{(2)} A X_{k+1} & =A_{T, S}^{(2)} A\left[X_{k}+Z_{k+1} R_{k}\right], \\
& =A_{T, S}^{(2)} A X_{k}+A_{T, S}^{(2)} A X_{k} R_{k}+A_{T, S}^{(2)} A X_{k} R_{k}^{2}, \\
& =X_{k}+X_{k} R_{k}+X_{k} R_{k}^{2}, \\
& =X_{k}+\left(X_{k}+X_{k} R_{k}\right) R_{k}, \\
& =X_{k}+Z_{k+1} R_{k}, \\
& =X_{k+1} .
\end{aligned}
$$

This proves that, (i) holds for all $k \geq 0$. Next, (ii) follows for $k=0$ from Remark 2.2 and Lemma 2.2 as

$$
A A_{T, S}^{(2)} A X_{0}=P_{A T, S} A X_{0}=A X_{0}
$$

Suppose $A A_{T, S}^{(2)} A X_{k}=A X_{k}$ holds for some positive integer $k$. To show that it holds for $k+1$, we consider

$$
\begin{aligned}
A A_{T, S}^{(2)} A X_{k+1} & =A A_{T, S}^{(2)} A\left[X_{k}+Z_{k+1} R_{k}\right], \\
& =A A_{T, S}^{(2)} A X_{k}+A A_{T, S}^{(2)} A X_{k} R_{k}+A A_{T, S}^{(2)} A X_{k} R_{k}^{2}, \\
& =A X_{k}+A X_{k} R_{k}+A X_{k} R_{k}^{2}, \\
& =A\left[X_{k}+Z_{k+1} R_{k}\right], \\
& =A X_{k+1} .
\end{aligned}
$$

Thus, (ii) holds for all $k \geq 0$.

Theorem 2.1 Let the initial approximation $X_{0} \in \mathbb{C}^{n \times m}$ be such that $R\left(X_{0}\right) \subset T$ and $X_{0}=\beta G$ for non-zero real scalar $\beta$ and $G \in \mathbb{C}^{n \times m}$ such that $R(G) \subseteq T$ and $N(G) \supseteq S$. Starting with $X_{0}$, the iterative method given by (3)-(4) leads to a sequence $\left\{X_{k}\right\}$ converging to

$$
X_{\infty}=A_{T, S}^{(2)}=X_{0}\left(I-F_{0}\right)^{-1},
$$

if and only if $\rho\left(F_{0}\right)=\rho\left(P_{A T, S}-A X_{0}\right)<1$. Further, let $\left\|P_{A T, S}-A X_{0}\right\|=q<1$, then $X_{k}$ has the error estimation

$$
\left\|X_{k+1}-A_{T, S}^{(2)}\right\| \leq q^{3^{k}}(1-q)^{-1}\left\|X_{0}\right\| .
$$

Proof Denote the residual matrix as, $F_{k}=P_{A T, S}-A X_{k}=$ $A A_{T, S}^{(2)}-A X_{k}$. Using (3)-(4), Lemma 2.2 and Remark 2.2, we get

$$
\begin{aligned}
F_{k+1} & =P_{A T, S}-A X_{k+1}, \\
& =A A_{T, S}^{(2)}-A\left(X_{k}+Z_{k+1} R_{k}\right), \\
& =A A_{T, S}^{(2)}-\left(A X_{k}\right)^{3}-3 A X_{k}+3\left(A X_{k}\right)^{2}, \\
& =\left(A A_{T, S}^{(2)}-A X_{k}\right)^{3} .
\end{aligned}
$$

This gives,

$$
F_{k+1}=F_{k}^{3}=F_{0}^{3^{k+1}}=\left(P_{A T, S}-A X_{0}\right)^{3^{k+1}} .
$$

Since, $\rho\left(P_{A T, S}-A X_{0}\right)<1$, this gives $X_{k} \rightarrow A_{T, S}^{(2)}$ as $k \rightarrow \infty$ for any $X_{0}=\beta G$. Converse part of this theorem is easy to prove, hence omitted. Next, from Lemma 2.2 and (4) we get,

$$
X_{k+1}=X_{k}+Z_{k+1}\left(P_{A T, S}-A X_{k}\right) .
$$

This implies that

$$
X_{k+1}=X_{0}\left[I+\left(P_{A T, S}-A X_{0}\right)+\ldots\left(P_{A T, S}-A X_{0}\right)^{3^{k+1}-1}\right] .
$$

Taking $k \rightarrow \infty$ in (9) and using $\rho\left(P_{A T, S}-A X_{0}\right)<1$, we get

$$
X_{\infty}=A_{T, S}^{(2)}=X_{0}\left(I-\left(P_{A T, S}-A X_{0}\right)\right)^{-1}=X_{0}\left(I-F_{0}\right)^{-1} .
$$

Now, to get the error estimates, from (9) and (10), we have

$$
\begin{aligned}
X_{k}-A_{T, S}^{(2)} & =-X_{0}\left[\left(P_{A T, S}-A X_{0}\right)^{3^{k}}+\left(P_{A T, S}-A X_{0}\right)^{3^{k+1}}+\ldots\right] \\
& =-X_{0}\left(P_{A T, S}-A X_{0}\right)^{3^{k}}\left[I+\left(P_{A T, S}-A X_{0}\right)+\ldots\right]
\end{aligned}
$$

Now taking norm on both the sides of above equation and using $\left\|P_{A T, S}-A X_{0}\right\|=q<1$, we obtain

$$
\left\|X_{k}-A_{T, S}^{(2)}\right\| \leq\left\|X_{0}\right\| q^{3^{k}}\left[1+q+q^{2}+\ldots\right], \leq q^{3^{k}}(1-q)^{-1}\left\|X_{0}\right\| .
$$

This completes the proof. Following lemma describes the relationship between the error matrix $E_{k}=A_{T, S}^{(2)}-X_{k}$ and the residual matrix $F_{k}=P_{A T, S}-A X_{k}$.

Lemma 2.4 Let $A \in \mathbb{C}^{m \times n}$, then we have

$$
\left\|E_{k+1}\right\| \leq\left\|A_{T, S}^{(2)}\right\|\left\|F_{k}^{3}\right\| .
$$

Proof Denote $E_{k}=A_{T, S}^{(2)}-X_{k}$ as the error matrix for computing $A_{T, S}^{(2)}$, then we have,

$$
A E_{k+1}=A A_{T, S}^{(2)}-A X_{k+1}=F_{k+1}=F_{k}^{3} .
$$

Using (12) and Remark 2.2, we get

$$
\begin{aligned}
\left\|E_{k+1}\right\| & =\left\|X_{k+1}-A_{T, S}^{(2)}\right\|, \\
& =\left\|A_{T, S}^{(2)} A X_{k+1}-A_{T, S}^{(2)} A A_{T, S}^{(2)}\right\|, \\
& \leq\left\|A_{T, S}^{(2)}\right\|\left\|A E_{k+1}\right\|, \\
& \leq\left\|A_{T, S}^{(2)}\right\|\left\|F_{k}^{3}\right\| .
\end{aligned}
$$

This completes the proof.

Remark 2.3 Using (8), inequality (11) can be further simplified as

$$
\left\|E_{k+1}\right\| \leq\left\|A_{T, S}^{(2)}\right\|\left\|F_{k}^{3}\right\| \leq\left\|A_{T, S}^{(2)}\right\|\left\|F_{0}\right\|^{3^{k+1}} .
$$

Since $\rho\left(F_{0}\right)<1$, from (13) we get $\left\|E_{k+1}\right\| \rightarrow 0$ as $k \rightarrow \infty$. This leads to the conclusion that iterative method (3)-(4) converges to $A_{T, S}^{(2)}$ with the third order of convergence. 
Remark 2.4 The convergence of the iteration (3)-(4) requires $\rho\left(F_{0}\right)=\rho\left(P_{A T, S}-A X_{0}\right)<1$. However, this cannot be directly checked since it involves the unknown $A_{T, S}^{(2)}$. To verify this, we need an equivalent convergence condition given in Theorem 2.2 which does not involve $A_{T, S}^{(2)}$.

Following lemma from [23] will be needed to prove our further results.

Lemma 2.5 If $P \in \mathbb{C}^{n \times n}$ and $S \in \mathbb{C}^{n \times n}$ satisfy $P=P^{2}$ and $P S=S P$ then $\rho(P S) \leq \rho(S)$.

Proof Suppose that $\lambda$ is an eigenvalue of $P S$ and $x$ is a corresponding vector such that $P S x=\lambda x$. Then, $\lambda P x=\lambda x$, which implies that $\lambda=0$ or $P x=x$. If $P x=x$, then $S x=S P x=P S x=\lambda x$, i.e. $\lambda$ is an eigenvalue of $S$. Hence, the set of eigenvalues of $P S$ is the subset of the set of eigenvalues of $S$ union $\{0\}$, which implies that $\rho(P S) \leq \rho(S)$.

Theorem 2.2 For all non-negative eigenvalues of $A G$, we have $\rho\left(P_{A T, S}-A X_{0}\right)<1$ if

$$
\max _{1 \leq i \leq r}\left|1-\beta \lambda_{i}(A G)\right|<1 .
$$

Proof Denote $P=A A_{T, S}^{(2)}$ and $S=I-A X_{0}$. Then from Lemmas 2.2 and 2.3, we get

$$
\begin{aligned}
P S & =A A_{T, S}^{(2)}\left(I-A X_{0}\right)=A A_{T, S}^{(2)}-A A_{T, S}^{(2)} A X_{0}=A A_{T, S}^{(2)}-A X_{0}, \\
& =\left(I-A X_{0}\right) A A_{T, S}^{(2)}=A A_{T, S}^{(2)}-A X_{0} A A_{T, S}^{(2)}=A A_{T, S}^{(2)}-A X_{0}=S P .
\end{aligned}
$$

It is clear that $P^{2}=P$. Now from Lemma 2.5 , we have

$$
\begin{aligned}
\rho\left(P_{A T, S}-A X_{0}\right) & =\rho\left(A A_{T, S}^{(2)}-A X_{0}\right) \leq \rho\left(I-A X_{0}\right) \\
& =\max _{1 \leq i \leq r}\left|1-\beta \lambda_{i}(A G)\right|<1
\end{aligned}
$$

This completes the proof.

Corollary 2.1 Convergence condition $\max _{1 \leq i \leq r} \mid 1-\beta \lambda_{i}$ $(A G) \mid<1$ is equivalent to the following condition

$$
0<\beta<\frac{2}{\lambda_{\max }(A G)} .
$$

Proof The proof is obvious and hence not given here.

\section{Two-step accelerated iterative method for outer inverse}

The purpose of this section is to further accelerate the iterative method (3)-(4) described in section 2. Following the technique proposed in [26] for accelerating the convergence speed of Newtons' method, we can define our accelerated iterative method for approximating $A_{T, S}^{(2)}$ for $k=$ $0,1,2, \ldots$ by

$$
\begin{gathered}
Z_{k+1}=\gamma_{k+1}\left(X_{k}+X_{k} R_{k}\right), \\
X_{k+1}=X_{k}+Z_{k+1} R_{k},
\end{gathered}
$$

where, $X_{0}$ is taken as discussed in section $2, R_{k}=I-A X_{k}$ and $\gamma_{k+1}$ is the acceleration parameter. It should be noted that the acceleration parameter $\gamma_{k+1}$ is selected so that it minimizes a bound on the maximum distance of any nonzero singular values of $X_{k+1} A$ from 1 . Let bounds $\sigma_{\min }$ and $\sigma_{\max }$ on the singular values of $A$ are known such that

$$
\sigma_{\min } \leq \sigma_{r}^{2} \leq \sigma_{1}^{2} \leq \sigma_{\max } .
$$

In this case, every eigenvalue of $G A$ lies in interval $\left[\sigma_{\min }, \sigma_{\max }\right]$. Assuming that this interval has a non-zero length, otherwise, $A$ is a scalar multiple of a unitary matrix and $G A$ is its inverse. Taking $\rho_{j}^{(0)}=\frac{2 \sigma_{j}^{2}}{\sigma_{\min }+\sigma_{\max }}$ and $\rho_{j}^{(k+1)}=$ $\gamma_{k+1}\left(2-\rho_{j}^{(k)}\right) \rho_{j}^{(k)}$ denotes lower and upper bounds on the singular values of $\beta G A$. The values given by

$$
\underline{\rho}^{(0)}=\frac{2 \sigma_{\min }}{\sigma_{\min }+\sigma_{\max }},
$$

and

$$
\bar{\rho}^{(0)}=\frac{2 \sigma_{\max }}{\sigma_{\min }+\sigma_{\max }} .
$$

are used for the acceleration parameters $\gamma_{k+1}, k>0$. Now, we can define

$$
\gamma_{k+1}=\bar{\rho}^{(k+1)}=\frac{2}{1+\left(2-\underline{\rho}^{(k)}\right) \underline{\rho}^{(k)}},
$$

as an acceleration parameter and an upper bound of $\left\{\rho_{j}^{(k+1)}\right\}$, and

$$
\underline{\rho}^{(k+1)}=\gamma_{k+1}\left(2-\underline{\rho}^{(k)}\right) \underline{\rho}^{(k)},
$$

as a lower bound on $\left\{\rho_{j}^{(k+1)}\right\}$. The definitions (18) and (19) gives for all $1 \leq j \leq r$, and $k \geq 1$,

$$
\underline{\rho}^{(k)} \leq \rho_{j}^{(k)} \leq \bar{\rho}^{(k)} .
$$

From (20) and (21), we get

$$
\underline{\rho}^{(k)}=2-\bar{\rho}^{(k)} .
$$

Likewise, it is easy to prove that $\rho_{j}^{(k)} \leq \bar{\rho}^{(k)}$. Finally, if $0 \leq \rho^{(k)} \leq \rho_{j}^{(k)} \leq\left(2-\rho^{(k)}\right)$ holds, then we have $\left(2-\underline{\rho}^{(k)}\right) \underline{\rho}^{(k)} \leq\left(2-\rho_{j}{ }^{(k)}\right) \rho_{j}{ }^{(k)} \leq 1$. Except for the last few iterations before convergence, $\underline{\rho}^{(k)} \ll 1$, which implies $\gamma_{k+1} \approx 2$ and $\rho_{j}{ }^{(k+1)} \approx 4 \rho_{j}{ }^{(k)}$.

Following algorithm gives the implementation of accelerated iterative method (16)-(17) to compute the outer inverse $A_{T, S}^{(2)}$ for matrix $A$. 
Algorithm 3.1 (Algorithm for computing $A_{T, S}^{(2)}$ from iterative method (16)-(17))

1: Initialization: Input matrix $A, X_{0}=\beta G, 0<\beta<\frac{2}{\lambda_{\max }(A G)}$ and $\rho^{(0)}$.

2: Perform the following steps $3,4,5$ until the termination criteria are satisfied.

3: $\quad \gamma_{k+1}=\frac{2}{1+\left(2-\rho^{(k)}\right) \rho^{(k)}}$.

4: $\quad Z_{k+1}=\gamma_{k+1}\left(X_{k}+X_{k} R_{k}\right)$.

5: Compute $X_{k+1}=X_{k}+Z_{k+1} R_{k}$.

6: $\quad$ Return the output $A_{T, S}^{(2)}$.

The proposed two-step accelerated iteration(16)-(17) and general two-step iteration (3)-(4) require three matrixmatrix multiplications per iteration. The only additional cost in (16)-(17) is the computation of $\gamma_{k+1}=\bar{\rho}^{(k+1)}$ which can be done with few operations by their formulas.

\section{Numerical experiments}

Numerical experiments involving singular square, rectangular, random matrices and a sparse matrix obtained by discretization of the Poisson's equation are solved. Iterations count (NN), computational time in seconds (CT) and the error bounds are used to measure the performance of our methods. As a case studies of the $A_{T, S}^{(2)}$, the $A^{\dagger}$ and $A_{g}$ are used in the numerical examples.

Example 4.1 ([10]) (Academical test) Let $A$ be the rectangular matrix of order $(3 \times 4)$ given by

$$
A=\left(\begin{array}{llll}
1 & 0 & 0 & -6 \\
2 & 6 & 0 & -6 \\
7 & 8 & 9 & -6
\end{array}\right)
$$

Starting with $X_{0}=\beta A^{*}$, where $G=A^{*}$, the proposed iteration method converges to the outer inverse $A_{R\left(A^{*}\right), N\left(A^{*}\right)}^{(2)}=A^{\dagger}$ given by

$$
A^{\dagger}=\left(\begin{array}{ccc}
\frac{28}{1931} & -\frac{143}{3862} & \frac{84}{1931} \\
-\frac{653}{3862} & \frac{1335}{7724} & -\frac{14}{1931} \\
\frac{57}{1931} & -\frac{249}{1931} & \frac{171}{1931} \\
-\frac{1903}{11586} & -\frac{143}{23172} & \frac{14}{1931}
\end{array}\right)
$$

where $\beta=\frac{1}{\|A\|^{2}}$, which satisfies the convergence condition $0<\beta<\frac{2}{\rho\left(A^{*} A\right)}$, discussed in Corollary 2.1. The stopping criteria given by $\left\|X_{k} A X_{k}-X_{k}\right\|_{2} \leq \epsilon=10^{-1000}$ with matrix 2-norm is used for error estimates. To clearly demonstrate the convergence behavior of the proposed method, we used 1000 fixed point digits in our computations analysis by using MATLAB's multiprecision package MATLAB vpa. The NN and estimation of error bounds obtained by accelerated method given by (16)-(17) compared with those obtained by Newton's method (1) and the method (2), for $p=3$ to compute $A^{\dagger}$ are summarized in tables 1,2 and 3 , respectively. It can be observed that proposed method converges considerably faster in comparison to other methods.

Example 4.2 Let $A$ be a randomly generated rectangular matrix of order $1000 \times 500$ generated by MATLAB function rand(1000, 500). The condition number of generated matrices is of the order $10^{2}$ and its entries are from the uniform distribution $U(0,1)$.

Starting with $X_{0}=\beta G$, where $G=A^{*}$ the proposed iteration method converges to the outer inverse $A_{R\left(A^{*}\right), N\left(A^{*}\right)}^{(2)}=A^{\dagger}$. The choice of $\beta$ is taken such that the

Table 1. Comparison results for error norm $E_{k}=\left\|A^{\dagger}-X_{k}\right\|_{2}$ for computing $A^{\dagger}$.

\begin{tabular}{lccc}
\hline NN & Method (1) & Method (2) & Method (16)-(17) \\
\hline 6 & $8.0750 \mathrm{e}-002$ & $1.5761 \mathrm{e}-005$ & $1.4406 \mathrm{e}-006$ \\
7 & $2.2108 \mathrm{e}-002$ & $4.5011 \mathrm{e}-014$ & $3.4370 \mathrm{e}-017$ \\
8 & $1.6572 \mathrm{e}-003$ & $1.0484 \mathrm{e}-039$ & $4.6674 \mathrm{e}-049$ \\
9 & $9.3120 \mathrm{e}-006$ & $1.3246 \mathrm{e}-116$ & $1.1689 \mathrm{e}-144$ \\
10 & $2.9401 \mathrm{e}-010$ & $2.6717 \mathrm{e}-347$ & $7.4999 \mathrm{e}-358$ \\
\hline
\end{tabular}

Table 2. Comparison results for residual norm $F_{k}=\| A A^{\dagger}-$ $A X_{k} \|_{2}$ for computing $A^{\dagger}$.

\begin{tabular}{lccc}
\hline NN & Method (1) & Method (2) & Method (16)-(17) \\
\hline 6 & $2.7379 \mathrm{e}+001$ & $5.3440 \mathrm{e}-005$ & $4.8845 \mathrm{e}-006$ \\
7 & $7.4960 \mathrm{e}-002$ & $1.5261 \mathrm{e}-013$ & $1.1653 \mathrm{e}-016$ \\
8 & $5.6190 \mathrm{e}-003$ & $3.5545 \mathrm{e}-039$ & $1.5825 \mathrm{e}-048$ \\
9 & $3.1573 \mathrm{e}-005$ & $4.4911 \mathrm{e}-116$ & $3.9632 \mathrm{e}-144$ \\
10 & $9.9685 \mathrm{e}-010$ & $9.0584 \mathrm{e}-347$ & $2.5429 \mathrm{e}-357$ \\
\hline
\end{tabular}

Table 3. Comparison results for norm $\left\|X_{k} A X_{k}-X_{k}\right\|_{2}$ for computing $A^{\dagger}$.

\begin{tabular}{lccc}
\hline NN & Method (1) & Method (2) & Method (16)-(17) \\
\hline 6 & $5.8642 \mathrm{e}-002$ & $1.5761 \mathrm{e}-005$ & $1.4406 \mathrm{e}-006$ \\
7 & $2.0451 \mathrm{e}-002$ & $4.5011 \mathrm{e}-014$ & $3.4370 \mathrm{e}-017$ \\
8 & $1.6479 \mathrm{e}-003$ & $1.0484 \mathrm{e}-039$ & $4.6674 \mathrm{e}-049$ \\
9 & $9.3117 \mathrm{e}-006$ & $1.3246 \mathrm{e}-116$ & $1.1689 \mathrm{e}-144$ \\
10 & $2.9401 \mathrm{e}-010$ & $2.6717 \mathrm{e}-347$ & $7.4999 \mathrm{e}-358$ \\
\hline
\end{tabular}




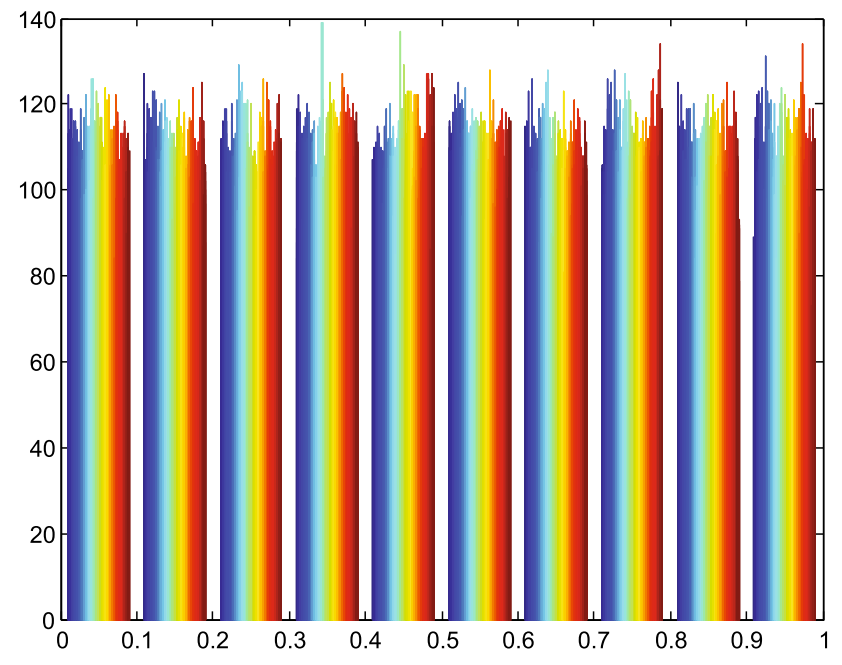

Figure 1. Histogram to visualize a distribution of numbers in matrix $A$ for Example 4.2.

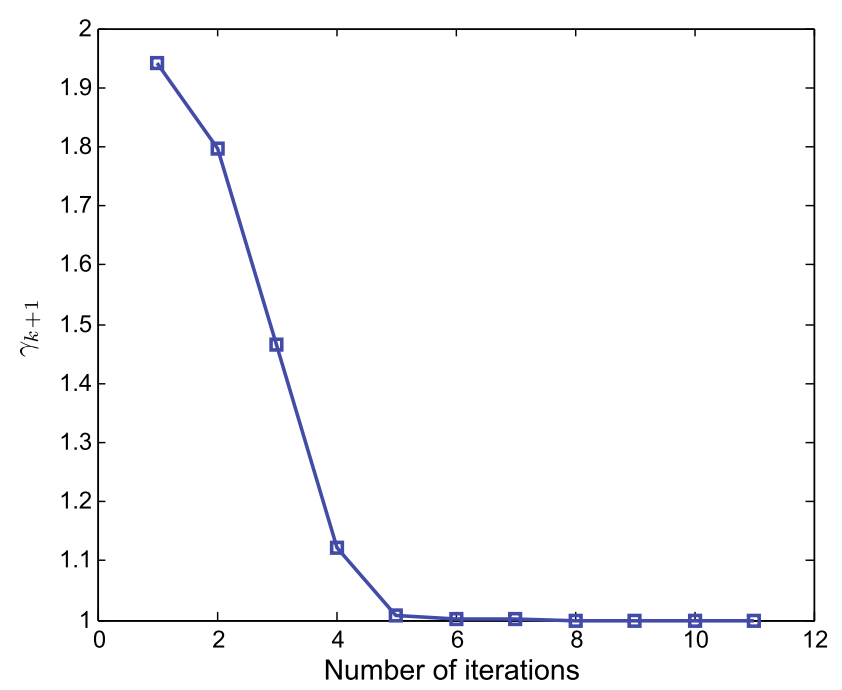

Figure 2. Variation of parameter $\gamma_{k+1}$ for Example 4.2.

convergence condition $0<\beta<\frac{2}{\rho\left(A^{*} A\right)}$ is satisfied. The termination criteria are given as $\max \left\{\left\|A X_{k} A-A\right\|_{2}, \| X_{k} A X_{k}\right.$ $\left.-X_{k}\left\|_{2},\right\|\left(A X_{k}\right)^{*}-A X_{k}\left\|_{2}, \quad\right\|\left(X_{k} A\right)^{*}-X_{k} A \|_{2}\right\} \leq \epsilon=10^{-7}$. Figure 1 gives the visualization of the distribution of numbers from the uniform distribution $U(0,1)$ considered in the matrix $A$. Variation of the acceleration parameter $\gamma_{k+1}$ with respect to number of iterations is shown in figure 2. It can be observed that $\gamma_{k+1} \in[1,2]$, which also confirms the theoretical observation discussed in section 3 . Table 4 compares the error bounds and $\mathrm{NN}$ by applying the proposed accelerated method (16)-(17), and iterative methods (1) and (2). Further, figure 3 shows the comparison of computational time. Note that $x$-axis in figure 3 represents the number of times random matrices are selected which is 30 , whereas $y$-axis represents the computational time in seconds. Again, it can be observed that the proposed method (16)-(17) converges faster than the methods (1) and (2) and gives improved results.

Example 4.3 Let $A$ be a randomly generated singular matrix of the order $600 \times 600$. The rank and index $j$ of generated matrix are 300 and 1, respectively.

Starting with $X_{0}=\beta G$, where $G=A^{j}=A$, the proposed iteration method converges to the outer inverse $A_{R(A), N(A)}^{(2)}=A_{g}$. The choice of $\beta$ is taken such that the convergence condition $0<\beta<\frac{2}{\rho\left(A^{j} A\right)}$ is satisfied. The termination criteria are given by $\max \left\{\left\|A X_{k} A-A\right\|_{2}\right.$, $\left.\left\|X_{k} A X_{k}-X_{k}\right\|_{2},\left\|A X_{k}-X_{k} A\right\|_{2}\right\} \leq \epsilon=10^{-7}$. Figure 4 gives the visualization of the distribution of numbers considered in the matrix $A$. It should be noted that entries of matrix $A$ does not follow any particular distribution. Variation of the acceleration parameter $\gamma_{k+1}$ with respect to number of iterations is shown in figure 5. It can be observed that $\gamma_{k+1} \in[1,2]$, which again confirms the theoretical observation discussed in section 3 . Table 5 gives comparison results in terms of error estimates obtained by applying the proposed accelerated method (16)-(17), iterative methods (1), (2) and method from [27]. Additionally, comparison results of computational time and number of iterations obtained by applying these methods are shown in figures 6 and 7, respectively. $x$-axis in figures 6 and 7 represents the number of times random matrices are selected which is 30 , whereas $y$-axis represents the computational time in seconds and number of iterations, respectively. From table 5, it can be observed that error estimates obtained by (16)-(17) is almost same as those obtained by methods (1) and (2). However, method from [27] was not able to give correct results due to large error for $\left\|A X_{k} A-A\right\|_{2}$. On the other hand, accelerated method outperforms methods (1) and (2)

Table 4. Comparison results for computing $A^{\dagger}$.

\begin{tabular}{lccc}
\hline Performance measures & Method (1) & Method (2) & Method (16)-(17) \\
\hline $\mathrm{NN}$ & 19 & 12 & 9 \\
$\left\|A X_{k} A-A\right\|_{2}$ & $5.1492 \mathrm{e}-13$ & $4.2628 \mathrm{e}-13$ & $4.1733 \mathrm{e}-13$ \\
$\left\|X_{k} A X_{k}-X_{k}\right\|_{2}$ & $2.3421 \mathrm{e}-14$ & $1.1294 \mathrm{e}-15$ & $4.8129 \mathrm{e}-16$ \\
$\left\|\left(A X_{k}\right)^{*}-A X_{k}\right\|_{2}$ & $5.4890 \mathrm{e}-14$ & $4.8866 \mathrm{e}-14$ & $4.3010 \mathrm{e}-14$ \\
$\left\|\left(X_{k} A\right)^{*}-X_{k} A\right\|_{2}$ & $4.6644 \mathrm{e}-14$ & $3.7960 \mathrm{e}-14$ & $3.4129 \mathrm{e}-14$ \\
\hline
\end{tabular}




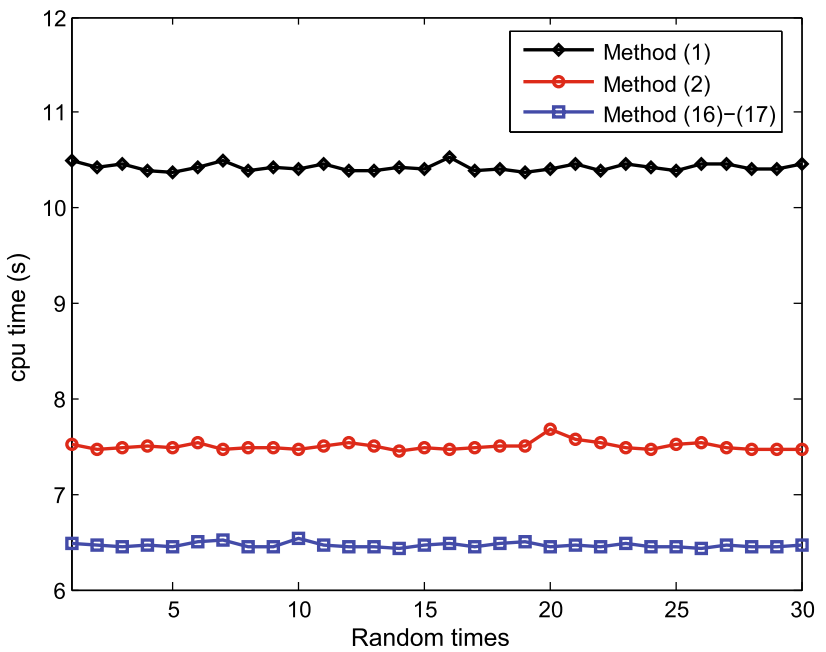

Figure 3. Comparison of CPU time for Example 4.2.

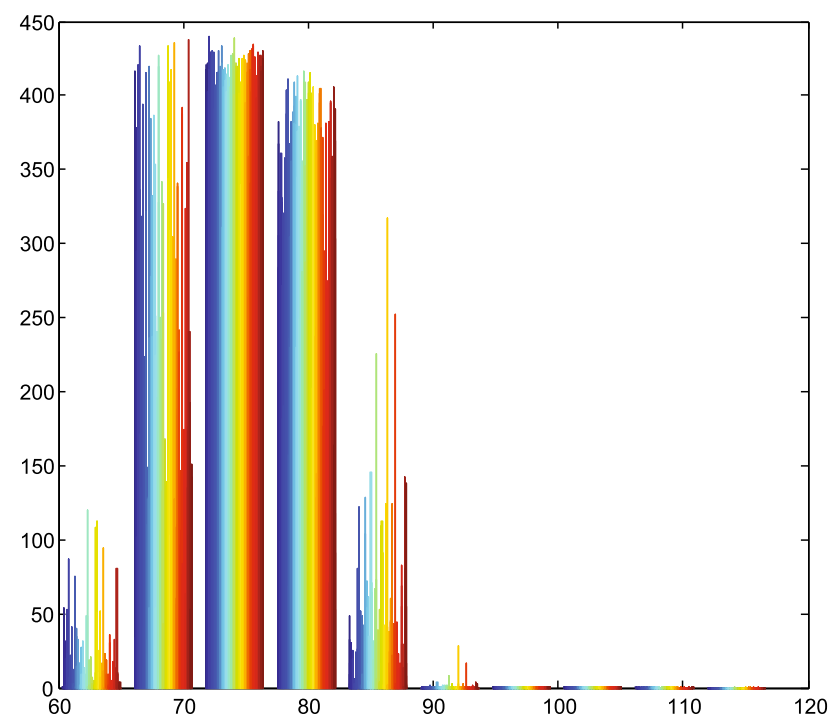

Figure 4. Histogram to visualize a distribution of numbers in matrix $A$ for Example 4.3.

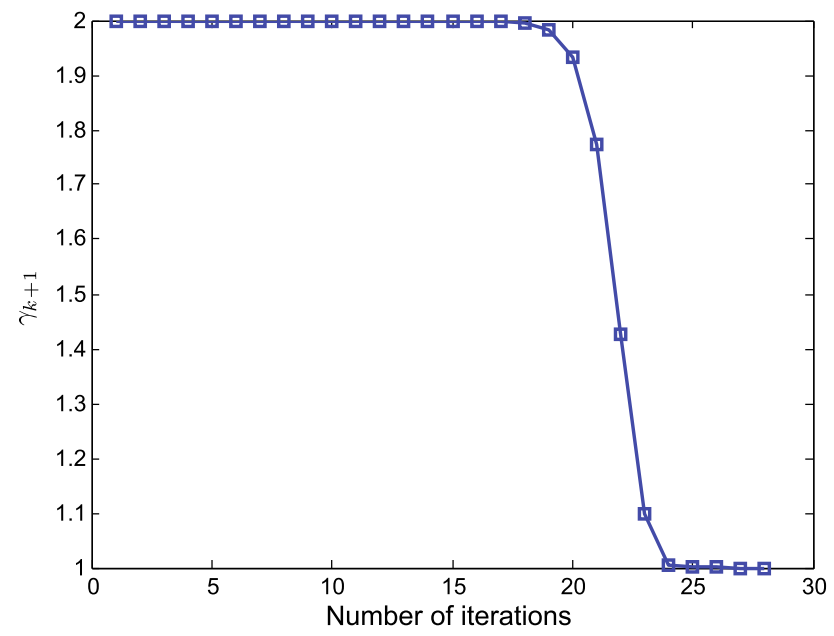

Figure 5. Variation of parameter $\gamma_{k+1}$ for Example 4.3. in terms of the computational speed, shown by figures 6 and 7, respectively. Note that computational time and number of iterations taken by method from [27] for each random run was approximately $35 \mathrm{~s}$ and 100, respectively, so we have omitted that from the figures. Thus one can conclude that significantly better performance is achieved by accelerated method (16)-(17).

Example 4.4 [28] Consider a sparse singular symmetric matrix $A$ of index $j=1$, where the matrix $A$ is an $(n+$ $1)^{2} \times(n+1)^{2}$ arises from the 5-point central difference discretization of the following Poisson's equation

$$
\frac{\partial^{2} w}{\partial x^{2}}+\frac{\partial^{2} w}{\partial y^{2}}=c(p, q),(p, q) \in \Omega=(0,1) \times(0,1)
$$

and all Neumann boundary conditions given by

$$
\frac{\partial w}{\partial N}=g
$$

where $c$ and $g$ satisfy the compatibility condition $\int_{\Omega} c d x d y=\int_{\partial \Omega} g d s$. Taking mesh size $h=\frac{1}{n}$, for $n=35$, the matrix $A$ of order $1296 \times 1296$ is given by,

$$
A=\left(\begin{array}{cccccc}
\frac{1}{2} V_{n+1}-W_{n+1} & & & & & \\
-W_{n+1} & V_{n+1} & -W_{n+1} & & & \\
& \ddots & \ddots & \ddots & & \\
& & & & & \\
& & & -W_{n+1} & V_{n+1} & -W_{n+1} \\
& & & & -W_{n+1} & \frac{1}{2} V_{n+1}
\end{array}\right),
$$

where $I_{n}$ is an $(n+1) \times(n+1)$ identity matrix and $V_{n+1}$ and $W_{n+1}$ are $(n+1) \times(n+1)$ matrices given as follows

$$
\begin{aligned}
& V_{n+1}=\left(\begin{array}{cccccc}
2 & -1 & & & & \\
-1 & 4 & -1 & & & \\
& \ddots & \ddots & \ddots & & \\
& & & & & -1 \\
& & & & 4 & -1
\end{array}\right) \text {, } \\
& W_{n+1}=\left(\begin{array}{cccccc}
\frac{1}{2} & & & & & \\
& 1 & & & & \\
& \ddots & \ddots & \ddots & & \\
& & & & & \\
& & & & 1 & \\
& & & & & \frac{1}{2}
\end{array}\right)
\end{aligned}
$$

Starting with $X_{0}=\beta G$, where $G=A^{j}=A$, the proposed iteration method converges to $A_{R(A), N(A)}^{(2)}=A_{g}$, the group 
Table 5. Comparison results for error bounds for computing $A_{g}$.

\begin{tabular}{lcccr}
\hline Error bounds & Method from [27] & Method (1) & Method (2) & Method (16)-(17) \\
\hline$\left\|A X_{k} A-A\right\|_{2}$ & $4.4819 \mathrm{e}+04$ & $9.7926 \mathrm{e}-09$ & $5.47061 \mathrm{e}-09$ & $2.3926 \mathrm{e}-09$ \\
$\left\|X_{k} A X_{k}-X_{k}\right\|_{2}$ & $2.2326 \mathrm{e}-12$ & $5.4083 \mathrm{e}-11$ & $4.5776 \mathrm{e}-11$ & $1.1567 \mathrm{e}-11$ \\
$\left\|A X_{k}-X_{k} A\right\|_{2}$ & $3.2466 \mathrm{e}-22$ & $6.0302 \mathrm{e}-11$ & $5.9075 \mathrm{e}-11$ & $3.5234 \mathrm{e}-12$ \\
\hline
\end{tabular}

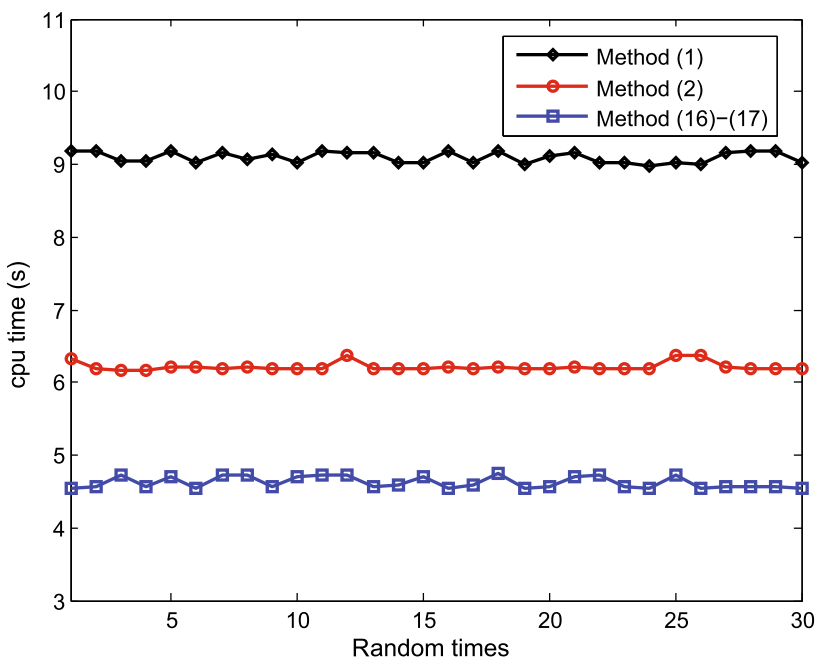

Figure 6. Comparison of CPU time for Example 4.3.

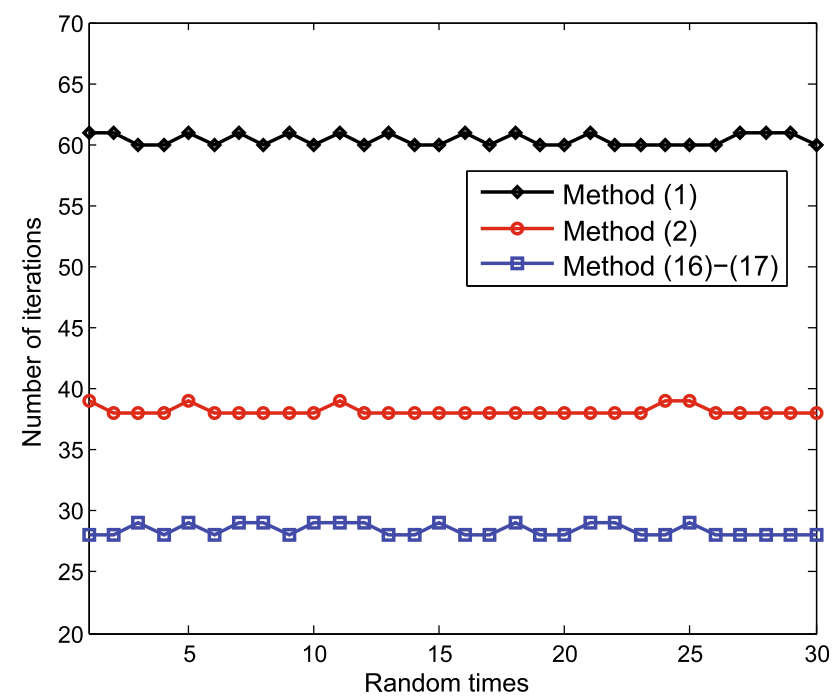

Figure 7. Comparison of number of iterations for Example 4.3.

inverse of $A$. The choice of $\beta$ is taken such that the convergence condition $0<\beta<\frac{2}{\rho\left(A^{j} A\right)}$ is satisfied. The termination criteria are same as taken in Example 4.3. The sparsity structure of matrix $A$ is illustrated in figure 8, where ' $n z$ ' stands for the number of non-zero entries of the matrix, which are 6300. Variation of the acceleration parameter $\gamma_{k+1}$ with respect to number of iterations are presented in

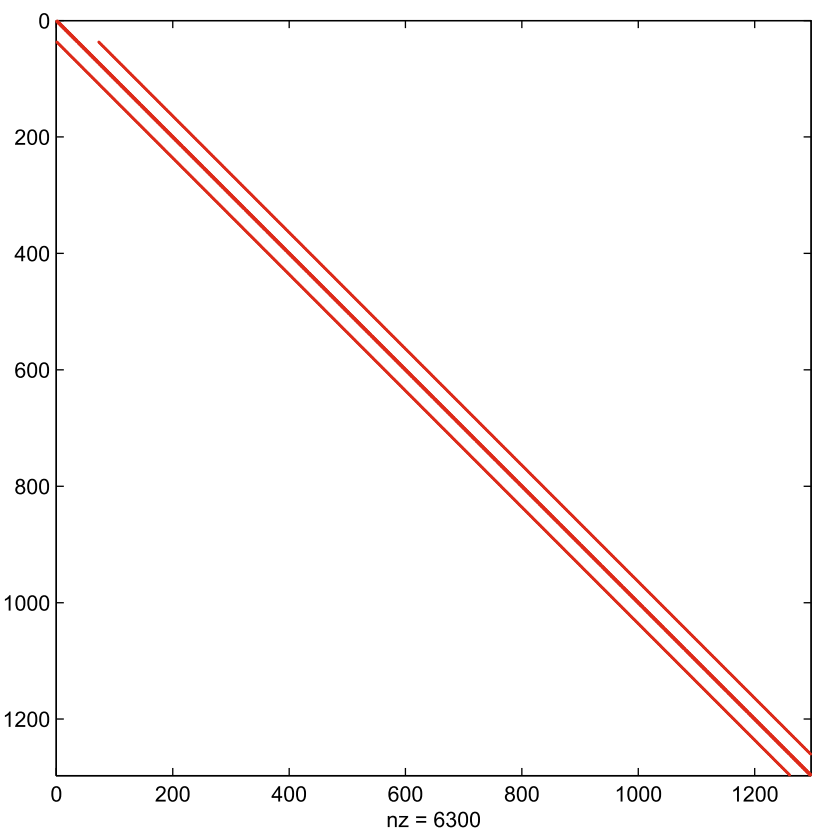

Figure 8. Sparsity structure of matrix for Example 4.4.

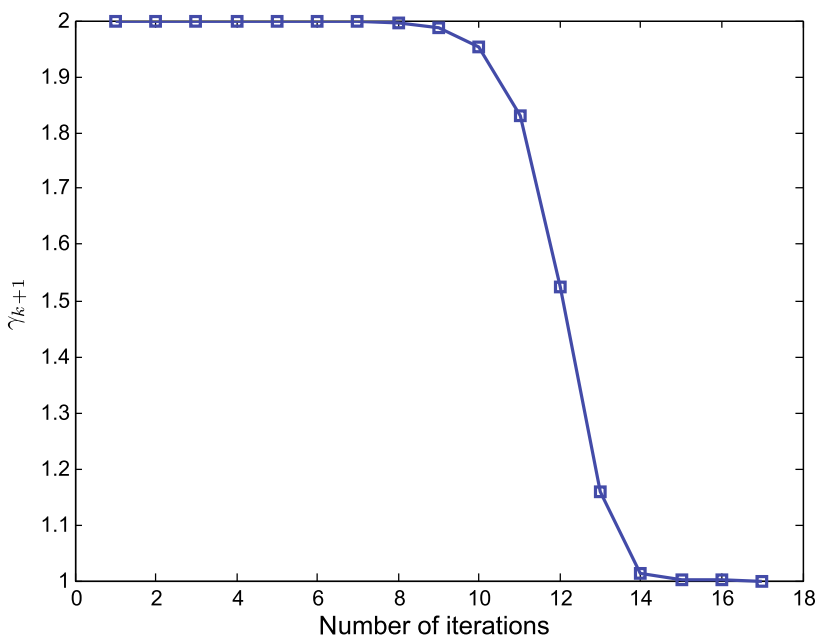

Figure 9. Variation of parameter $\gamma_{k+1}$ for Example 4.4.

figure 9. It can be observed that $\gamma_{k+1} \in[1,2]$, which confirms the theoretical observation discussed in section 3 . The performance measures CT, NN, and error bounds are compared with the methods (1), (2) as well as with the 
Table 6. Comparison results for CT, NN and error bounds for computing $A_{g}$.

\begin{tabular}{lcccc}
\hline Performance measures & Method from [27] & Method (1) & Method (2) & Method (16)-(17) \\
\hline CT & 183.5531 & 78.0728 & 52.1202 & 40.4580 \\
NN & 100 & 35 & 22 & 17 \\
$\left\|A X_{k} A-A\right\|_{2}$ & $1.95756 \mathrm{e}+00$ & $3.5475 \mathrm{e}-12$ & $7.5044 \mathrm{e}-12$ & $3.7460 \mathrm{e}-12$ \\
$\left\|X_{k} A X_{k}-X_{k}\right\|_{2}$ & $8.0522 \mathrm{e}-02$ & $5.2762 \mathrm{e}-09$ & $1.6572 \mathrm{e}-09$ & $4.2130 \mathrm{e}-09$ \\
$\left\|A X_{k}-X_{k} A\right\|_{2}$ & $2.8613 \mathrm{e}-15$ & $1.0772 \mathrm{e}-11$ & $1.2288 \mathrm{e}-11$ & $9.9853 \mathrm{e}-12$ \\
\hline
\end{tabular}

method studied in [27]. The results are tabulated in table 6 . Again it can be observed that accelerated method (16)-(17) takes very less computational time and number of iterations to achieve almost the same level of accuracy than the methods (1) and (2) and gives improved results.

\section{Conclusions}

A two-step iteration and its accelerated version for computing the outer inverse $A_{T, S}^{(2)}$ of an arbitrary matrix $A$ is described. It is based on the well-known Newton's method for approximating outer inverse of arbitrary matrix $A$. The convergence analysis along with the estimation of error bounds is derived. Numerical experiments involving singular square, rectangular, random matrices and a sparse matrix obtained by discretization of the Poisson's equation are solved. Iterations count (NN), computational time (CT) and the error bounds are used to measure the performance of our methods. As a case studies of the $A_{T, S}^{(2)}$, the Moore-Penrose inverse $A^{\dagger}$ and group inverse $A_{g}$ are used in the numerical examples. The results obtained from the accelerated iterative method are compared with those obtained by some of the existing methods. It is apparent that the accelerated iterative method converges considerably faster in comparison with other existing methods. Thus, both computational speed and accuracy are significantly improved by it.

The work of this paper can be extended in various directions. Some of the future works to be carried out can be listed as follows.

- As an application, one can modify the proposed iterative methods to approximate generalized inverses of structured matrices such as Toeplitz and Hankel matrices.

- One can also extend the proposed iterative to approximate the outer inverse $A_{T, S}^{(2)}$ of bounded linear operators $A$ between Banach spaces.

\section{Acknowledgments}

The authors thank the referees for their valuable comments which have improved the presentation of the paper.

\section{References}

[1] Petković M D and Petković M S 2015 Hyper-power methods for the computation of outerinverses. J. Comput. Appl. Math. 278: 110-118

[2] Stanimirović P S, Chountasis S, Pappas D and Stojanović I 2013 Removal of blur in images based on least squares solutions. Math. Methods Appl. Sci. 36(17): 2280-2296

[3] Nashed M Z 1976 Generalized inverse and applications. Academic Press, NewYork.

[4] Nashed M Z and Chen X 1993 Convergence of Newton-like methods for singular operator equations using outer inverses. Numer. Math. 66(1): 235-257

[5] Mosić D 2015 Reverse order laws for the generalized Drazin inverse in Banach algebras. J. Math. Analy. Appl. 429(1): 461-477

[6] Srivastava S and Gupta D K 2014 A higher order iterative method for $A_{T, S}^{(2)}$. J. Appl. Math. Comput. 46(1):147-168

[7] Kyrchei I I 2011 Determinantal representations of the Moore-Penrose inverse over the quaternion skew field and corresponding Cramer's rules. Linear Multilinear Algebra 59(4):413-431

[8] Smoktunowicz A and Wróbel I 2012 Numerical aspects of computing the Moore-Penrose inverse of full column rank matrices. BIT Numer. Math. 52(2): 503-524

[9] Stanimirović P S and Soleymani F 2014 A class of numerical algorithms for computing outer inverses. J. Comput. Appl. Math. 263: 236-245

[10] Soleymani F , Stanimirović P S and Haghani F K 2015 On hyperpower family of iterations for computing outer inverses possessing high efficiencies. Linear Algebra Appl. 484: 477-495

[11] Sheng X and Chen G 2011 New proofs of two representations and minor of generalized inverse $A_{T, S}^{(2)}$. Appl. Math. Comput. 217(13): 6309-6314

[12] Stanimirović P S, Pappas D, Katsikis V N and Stanimirović I P 2012 Full-rank representations of outer inverses based on the QR decomposition. Appl. Math. Comput. 218(20): 10321-10333

[13] Stanimirović I P and Tasić M B 2012 Computation of generalized inverses by using the $L D L^{*}$ decomposition. Appl. Math. Lett. 25(3): 526-531

[14] Stanimirović P S and Petković M D 2013 Gauss-Jordan elimination method for computing outer inverses. Appl. Math. Comput. 219(9): 4667-4679

[15] Stanimirović I P 2015 Computing $A_{T, S}^{(2)}$ inverses of Hermitian matrices via $L D L^{*}$ decomposition for a square matrix $A$. Linear Multilinear Algebra 63(8): 1553-1567 
[16] Ben-Israel A and Greville T N E 2003 Generalized inverses: Theory and applications, second ed. Springer, New York

[17] Chen Y and Chen X 2000 Representation and approximation of the outer inverse $A_{T, S}^{(2)}$ of a matrix A. Linear Algebra Appl. 308(1): 85-107

[18] Chen Y and Tan X 2005 Computing generalized inverses of matrices by iterative methods based on splittings of matrices. Appl. Math. Comput. 163(1): 309-325

[19] Petković M D and Stanimirović P S 2014 Two improvements of the iterative method for computing Moore-Penrose inverse based on Penrose equations. J. Comput. Appl. Math. 267: $61-71$

[20] Liu X, Jin H and Yu Y 2013 Higher-order convergent iterative method for computing the generalized inverse and its application to Toeplitz matrices. Linear Algebra Appl. 439(6): $1635-1650$

[21] Chen Y 1996 Iterative methods for computing the generalized inverses $A_{T, S}^{(2)}$ of a matrix A. Appl. Math. Comput. 75: 207-222

[22] Sheng X 2012 An iterative algorithm to compute the generalized inverse $A_{T, S}^{(2)}$ under the restricted inner product. Int. J. Math. Comput. Sci. 6: 900-904
[23] Stanimirović P S and CvetkovićIlić D S 2008 Successive matrix squaring algorithm for computing outer inverses. Appl. Math. Comput. 203(1): 19-29

[24] Srivastava S and Gupta D K 2015 An iterative method for solving general restricted linear equations. Appl. Math. Comput. 262: 344-353

[25] Srivastava S and Gupta D K 2014 A new representation for $A_{T, S}^{(2,3)}$. Appl. Math. Comput. 243: 514-521

[26] Pan V Y and Schreiber R 1991 An improved Newton iteration for the generalized inverse of a matrix. SIAM J. Sci. Stat. Comput. 12(5): 1109-1131

[27] Zhong J, Liu X, Zhou G and Yu Y 2012 A new iterative method for computing the Drazin inverse. Filomat 26(3): 597-606

[28] Zhang S, Oyanagi Y and Sugihara M 2000 Necessary and sufficient conditions for the convergence of Orthomin $(k)$ on singular and inconsistent linear systems. Numerishe Mathe. 87(2): 391-405 